\title{
Tyrosine kinase inhibitors improve parenchymal findings of liver cirrhosis in a patient exhibiting concomitant hepatocellular carcinoma and renal cell cancer
}

\author{
TULAY KUS $^{1}$, GOKMEN AKTAS ${ }^{1}$, ALPER SEVINC ${ }^{1}$, CEMIL OKTAY $^{2}$, \\ MEHMET EMIN KALENDER ${ }^{1}$ and CELALETDIN CAMCI ${ }^{1}$ \\ ${ }^{1}$ Division of Medical Oncology, Department of Internal Medicine, Gaziantep Oncology Hospital, \\ School of Medicine, Gaziantep University, Gaziantep TR-27310; ${ }^{2}$ Department of Radiology, \\ School of Medicine, Akdeniz University, Antalya TR-07100, Turkey
}

Received June 3, 2015; Accepted November 3, 2015

DOI: $10.3892 / \mathrm{mco} .2015 .677$

\begin{abstract}
Hepatocellular carcinoma (HCC) and renal cell cancer (RCC) are malignancies, which are chemotherapy resistant and fatal at the advanced stages. Previously developed tyrosine kinase inhibitors are used in the treatment of advanced stage disease. In the present case study, a patient using sunitinib for stage IV RCC presented with HCC following 2 years of treatment. A patient who exhibited Child-Pugh class $\mathrm{C}$ cirrhosis initially, exhibited a marked improvement of hepatocellular parenchyma findings following treatment with sunitinib. Sunitinib is suggested to have preventive effects on the pathogenesis of liver fibrosis and cirrhosis in vitro, via an anti-vascular endothelial growth factor and anti-platelet-derived growth factor mechanism. However, no clinical supportive study has been performed until now. Improvement of liver functions may be explained in this manner. Therefore, investigations are required with different doses of sunitinib and other tyrosine kinase inhibitors in order to evaluate the efficacy on treatment of cirrhosis progression.
\end{abstract}

\section{Introduction}

Hepatocellular carcinoma (HCC) is the second leading cause of cancer-associated mortality worldwide and is generally associated with hepatitis B or $\mathrm{C}$ viruses, which account for $80 \%$ of HCC (1). Cirrhosis is also a risk factor for HCC, irrespective of the etiology of the cirrhosis. The annual risk of developing

Correspondence to: Dr Tulay Kus, Division of Medical Oncology, Department of Internal Medicine, Gaziantep Oncology Hospital, School of Medicine, Gaziantep University, Yuzdortbinelli 104050, Gaziantep TR-27310, Turkey

E-mail: drtulaykus83@hotmail.com

Key words: hepatocellular carcinoma, renal cell cancer, sunitinib, sorafenib, cirrhosis
HCC among patients with cirrhosis is between 1 and 6\% (2). Despite performed resection and locoregional therapies, recurrence occurs often and is lethal. For this reason, in order to prevent early recurrence, the use of antiviral therapies is essential and also adjuvant treatment with tyrosine kinase inhibitors following curative modality is recommended in certain previous studies $(3,4)$. Using sorafenib in addition to transarterial chemoembolization (TAKE) is currently under investigation. However, if there is evidence of residual or recurrent tumor, sorafenib treatment following arterially directed therapies is appropriate (5).

Renal cell cancer (RCC) is a tumor with increasing incidence among patients with cancer. The 5 year survival rate of kidney cancer is $98.1 \%$ for localized disease and $12.3 \%$ for advanced disease. Following curative treatment of the localized disease, observation remains the standard of care. Patients with stage IV disease also may benefit from cytoreductive surgery (6). For advanced disease, targeted therapy with tyrosine kinase and vascular endothelial growth factor (VEGF) antibodies (e.g. sunitinib, sorafenib, pazopanib, axitinib, temsirolimus, everolimus and bevacizumab, in combination with interferon) are used sequentially (7-9).

In the present case, a patient presenting with liver cirrhosis demonstrated improvement in Child-Pugh scores following treatment with sunitinib for renal cell carcinoma. Sunitinib is an agent exhibiting improvements in liver cirrhosis findings at preclinical study (10). In this context, the present study can pave the way for designing future studies investigating the use of sunitinib in the treatment of liver cirrhosis. Also, sunitinib can be used for patients who have sorafenib intolerance in HCC with concomitant RCC treatment.

\section{Case report}

A 62-year-old male was diagnosed with Child-Pugh class C (score, 10) liver cirrhosis with chronic hepatitis B infection in 2005. Antiviral therapy with lamivudine was initiated following the diagnosis. In February 2007, the patient presented gross hematuria. Ultrasonography indicated a solid mass in the right kidney. Right radical nephrectomy 

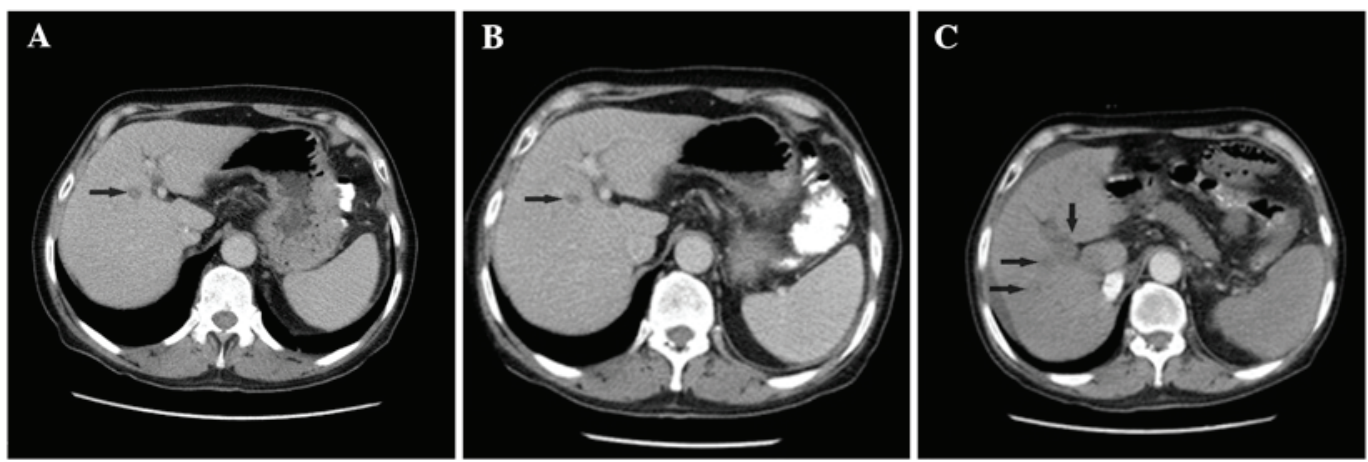

Figure 1. Abdominal computed tomography reveals a tissue mass in the liver. (A) In March 2012, a mass was observed in segment $4 \mathrm{~b}$ of the liver sized $1.5 \times 1 \mathrm{~cm}$. (B) In April 2013, minimal progression was detected. (C) In April 2014, progression of the lesion was detected during the procedure.

was performed on the patient and postoperative pathological examination confirmed clear-cell carcinoma (tumor $>7 \mathrm{~cm}$ in greatest dimension, limited to kidney with unknown lymph node status and unknown distant metastases; pT2NxMx). In March 2007, a computed tomography (CT) scan revealed multiple millimeter sized lung metastases. A lung metastasectomy was performed and lung metastasis was confirmed. The patient had an Eastern Cooperative Oncology Group performance status of 1 . Interferon treatment was administered for 6 months. Subsequently, temsirolimus treatment was initiated as a result of the progression of lung metastasis. This treatment was terminated due to inadequate hepatocellular function (Child-Pugh class C; score, 12). The patient was not examined for 2 years as a personal choice. During this examination, decompensation of liver functions with ascites was observed. The patient subsequently resumed antiviral therapy. In February 2010, the patient complained of fatigue and itching, and was evaluated as Child-Pugh class C (score, 10; controlled ascites with diuretics). A CT scan revealed progression of the lung metastasis, right kidney mass (size, $37 \times 28 \mathrm{~mm}$ ) and left surrenal mass (size, $5 \times 4.5 \mathrm{~cm}$ ). Sunitinib was administered at $50 \mathrm{mg}$ once daily on a standard 4 weeks on, 2 weeks off schedule of 6 week cycles. Following treatment with sunitinib for 6 months and no change in antiretroviral therapy, the hepatocellular parenchyma failure finding was improved unexpectedly to Child-Pugh class A (score, 5). During the treatment period, grade 2 thrombocytopenia and grade 2 anemia developed. Due to this toxicity, the dose was adjusted to $37.5 \mathrm{mg}$ once daily for 2 years. In March 2012, an abdominal CT scan revealed a mass in the segment $4 \mathrm{~b}$ of the liver (size, 1.5x1 cm; Fig. 1A). This lesion was closely monitored. In April 2013, a lesion biopsy was performed due to minimal progression (Fig. 1B). Pathological examination determined well differentiated HCC. The level of $\alpha$-fetoprotein (AFP) was $20 \mathrm{ng} / \mathrm{ml}$ and the patient was Child-Pugh class A. TAKE with doxorubicin was performed three times during this period (May-July 2013). Due to concomitant HCC and RCC, sunitinib treatment was modified to sorafenib. However, the patient refused this modification due to development grade III fatigue, asthenia and confusion with sorafenib treatment. Therefore, sunitinib treatment was continued. In April 2014, an abdominal CT scan revealed a necrotic mass in the location at which TAKE was performed, in right portal vein and para-aorto-cavale pathologic lymph nodes (Fig. 1C). The level of AFP was 4,548 ng/ml. Progression of RCC was not observed. Sunitinib treatment was discontinued as a result of HCC progression.

\section{Discussion}

The majority of patients with HCC are diagnosed at the advanced stages. The prognosis of advanced stage $\mathrm{HCC}$ is poor and the overall survival (OS) rate is $<5 \%$ (10). According to two large phase 3 randomized trials, the Asia-Pacific study and the SHARP study, sorafenib is an effective treatment in patients with advanced HCC $(11,12)$; however, it can be more toxic and lead to acute hepatocellular failure in the patients with Child-Pugh class B and C cirrhosis. Based on the National Comphrehensive Cancer Network guideline (http:// www.nccn.org/professionals/physician_gls/f_guidelines. asp), sorafenib is recommended as a category 1 option for patients with Child-Pugh class A liver function and as a category 2A option for patients with Child-Pugh class B liver function.

Sorafenib, an oral multi-kinase inhibitor, inhibits cell proliferation by targeting the Ras/mitogen-activated protein kinase signaling pathway at the level of the Raf kinase, and other tyrosine kinase receptors, including an antiangiogenic effect by inhibiting VEGF receptors 1-3, platelet-derived growth factor (PDGF) receptor $\beta$ and stem cell factor receptor. In two large phase III placebo-controlled studies, sorafenib at a dose of $800 \mathrm{mg} /$ day, significantly prolonged the OS rate in patients with advanced HCC, particularly with Child-Pugh class A cirrhosis (11,12). Therefore, sorafenib is currently considered as the standard treatment for patients with advanced HCC.

However, few patients respond to sorafenib and the majority of patients unavoidably eventuate with progression. In this instance, a phase II study was designed with sunitinib, which is an another tyrosine kinase inhibitor, for patients with advanced HCC. In the present study, sunitinib was administered at 50,37.5 and $25 \mathrm{mg} / \mathrm{day}$, based on toxicity dose adjustment. In the present study, the overall incidence of severe (grade, $\geq 3$ ) adverse events was $79.4 \%$. Toxicity was observed in the majority of the patients with Child-Pugh class B. The most common grade III or IV events included fatigue $(47 \%)$, nausea $(14.7 \%)$, liver failure $(14.7 \%)$ and 
hepatic encephalopathy (11.7\%) (10). A previous randomized phase III study compared the safety and efficiency of sunitinib and sorafenib. These drugs were administered as sunitinib at $37.5 \mathrm{mg}$ once daily or sorafenib at $400 \mathrm{mg}$ twice daily. Sunitinib was associated with more frequent and severe adverse events. The OS with sunitinib was inferior to sorafenib, however, it was similar in Asian and hepatitis B-infected patients (14).

In the present study, two findings are of great interest. Firstly, a patient without the diagnosis of HCC exhibited cirrhosis with Child-Pugh class C (score, 10) at the initiation of sunitinib treatment for RCC. Following sunitinib treatment, the Child-Pugh score regressed and decompensation of liver function with ascites was not observed. Majumder et al (15) performed in vitro experiments and the efficacy of Sunitinib for cirrhotic liver and its effect on angiogenesis were assessed. The development of HCC from malignant hepatocytes is frequently associated with intra-peritumoral accumulation of connective tissue arising from activated hepatic stellate cells (HSC). HSC also perform hepatic functions frequently ascribed to fibroblast cells, including deposition of collagen and migration in response to angiogenic stimuli. These cells have an active role in cirrhosis and carcinogenesis. In the present study, the receptor tyrosine kinase inhibitor, sunitinib, which is a PDGF and VEGF inhibitor, may inhibit activated HSC functions and angiogenesis. Therefore, it can prevent the progression of cirrhotic liver (15). In this circumstance, improvement of hepatic functions with sunitinib treatment may be explained in patients who have not yet developed HCC. Therefore, further studies are required with different doses of sunitinib and other tyrosine kinase inhibitors in order to evaluate the efficacy on treatment of hepatic parenchyma findings or cirrhosis progression, as observed in the present patient.

Secondly, sunitinib treatment was well tolerated by the patient. Although sorafenib was preferred, the patient refused this treatment due to the development of grade III fatigue, asthenia and confusion. The patient with concomitant stage IV RCC and HCC experienced no grade III-IV toxicity during sunitinib therapy. Sunitinib was demonstrated to have equal efficiency in hepatitis B patients, as indicated in the phase III study mentioned previously (14). Diagnosis of HCC secondary to hepatitis B in the present patient may explain the similar results of sorafenib treatment consistent with the literature mentioned previously. Sorafenib is the most efficient agent for HCC treatment. However, in the cases of sorafenib intolerance or concomitant HCC-RCC, sunitinib may be considered as an alternative treatment, since there is no other agent promising a proven efficiency.

\section{References}

1. Jemal A, Bray F, Center MM, Ferlay J, Ward E and Forman D: Global cancer statistics. CA Cancer J Clin 61: 69-90, 2011.

2. Ikeda K, Saitoh S, Koida I, Arase Y, Tsubota A, Chayama K, Kumada $\mathrm{H}$ and Kawanishi M: A multivariate analysis of risk factors for hepatocellular carcinogenesis: A prospective observation of 795 patients with viral and alcoholic cirrhosis Hepatology 18: 47-53, 1993.

3. Zhang W, Zhao G, Wei K, Zhang Q, Ma W, Song T, Wu Q, Zhang T, Kong D and Li Q: Adjuvant sorafenib reduced mortality and prolonged overall survival and post-recurrence survival in hepatocellular carcinoma patients after curative resection: A single-center experience. Biosci Trends 8: 333-338, 2014.

4. Wang SN, Chuang SC and Lee KT: Efficacy of sorafenib as adjuvant therapy to prevent early recurrence of hepatocellular carcinoma after curative surgery: A pilot study. Hepatol Res 44: 523-531, 2014.

5. Chung YH, Han G, Yoon JH, Yang J, Wang J, Shao GL, Kim BI, Lee TY and Chao Y: Interim analysis of START: Study in Asia of the combination of TACE (transcatheter arterial chemoembolization) with sorafenib in patients with hepatocellular carcinoma trial. Int J Cancer 132: 2448-2458, 2013.

6. Culp SH, Tannir NM, Abel EJ, Margulis V, Tamboli P, Matin SF and Wood CG: Can we better select patients with metastatic renal cell carcinoma for cytoreductive nephrectomy? Cancer 116: 3378-3388, 2010.

7. Albiges L, Kube U, Eymard JC, Schmidinger M, Bamias A, Kelkouli N, Mraz B, Florini S, Guderian G, Cattaneo A, et al: Everolimus for patients with metastatic renal cell carcinoma refractory to anti-VEGF therapy: Results of a pooled analysis of non-interventional studies. Eur J Cancer 51: 2368-2374, 2015.

8. Oudard S and Vano Y: The role of rechallenge with targeted therapies in metastatic renal-cell carcinoma. Curr Opin Urol 25: 402-410, 2015.

9. Eichelberg C, Vervenne WL, De Santis M, Fischer von Weikersthal L, Goebell PJ, Lerchenmüller C, Zimmermann U, Bos MM, Freier W, Schirrmacher-Memmel S, et al: SWITCH: A Randomised, Sequential, Open-label Study to Evaluate the Efficacy and Safety of Sorafenib-sunitinib Versus Sunitinibsorafenib in the Treatment of Metastatic Renal Cell Cancer. Eur Urol 68: 837-847, 2015.

10. Wilhelm SM, Adnane L, Newell P, Villanueva A, Llovet JM and Lynch M: Preclinical overview of sorafenib, a multikinase inhibitor that targets both Raf and VEGF and PDGF receptor tyrosine kinase signaling. Mol Cancer Ther 7: 3129-3140, 2008.

11. Llovet JM, Ricci S, Mazzaferro V, Hilgard P, Gane E, Blanc JF, de Oliveira AC, Santoro A, Raoul JL, Forner A, et al: Sorafenib in advanced hepatocellular carcinoma. N Engl J Med 359: 378-390, 2008.

12. Cheng AL, Kang YK, Chen Z, Tsao CJ, Qin S, Kim JS, Luo R, Feng J, Ye S, Yang TS, et al: Efficacy and safety of sorafenib in patients in the Asia-Pacific region with advanced hepatocellular carcinoma: A phase III randomised, double-blind, placebo-controlled trial. Lancet Oncol 10: 25-34, 2009.

13. Barone C, Basso M, Biolato M, Pompili M, Rufini V, Miele L, Basso M, De Gaetano AM, Castaldi P, Iaculli A, et al: A phase II study of sunitinib in advanced hepatocellular carcinoma. Dig Liver Dis 45: 692-698, 2013.

14. Cheng AL, Kang YK, Lin DY, Park JW, Kudo M, Qin S, Chung HC, Song X, Xu J, Poggi G, et al: Sunitinib versus sorafenib in advanced hepatocellular cancer: Results of a randomized phase III trial. J Clin Oncol 31: 4067-4075, 2013.

15. Majumder S, Piguet AC, Dufour JF and Chatterjee S: Study of the cellular mechanism of sunitinib mediated inactivation of activated hepatic stellate cells and its implications in angiogenesis. Eur J Pharmacol 705: 86-95, 2013. 